\title{
Association of Toll-like receptor (TLR) 3 \& 9 genes' polymorphism with Hepatitis C virus-specific cell-mediated immunity outcome among Egyptian healthcare workers
}

\author{
Sayed Abdelwahab ${ }^{1}$, Shaimaa Hamdy ${ }^{2}$, Ahmed Osman ${ }^{3}$, Zainab Zakaria ${ }^{4}$, Iman Galal ${ }^{5}$, \\ Maha Sobhy ${ }^{6}$, Mohamed Hashem ${ }^{7}$, Walaa Allam ${ }^{8}$, Mohamed Abdel-Samiee ${ }^{9}$, Eman \\ Rewisha $^{9}$, and Imam Waked ${ }^{9}$ \\ ${ }^{1}$ Minia University Faculty of Medicine \\ ${ }^{2}$ The Egyptian Holding Company for Biological Products and Vaccines \\ ${ }^{3}$ Cairo University Faculty of Science \\ ${ }^{4}$ Heliopolis University for Sustainable Development \\ ${ }^{5}$ The Egyptian Holding Company for Biological Products and Vaccines (VACSERA), \\ 6 The Egyptian Holding Company for Biological Products and Vaccines (VACSERA) \\ ${ }^{7}$ University of Maryland School of Medicine \\ ${ }^{8}$ Zewail City of Science and Technology - Zewail City Campus \\ ${ }^{9}$ Menoufia University National Liver Institute
}

June 17, 2020

\begin{abstract}
Variations in immune responses could define successful resistance to Hepatitis C Virus (HCV) infection. Toll-like receptors (TLR)-3 are innate detectors of dsRNA viruses while TLR9 recognizes bacterial and viral unmethylated CpG motifs. We previously reported that TLR3.rs3775290 "CC" genotype was associated with HCV chronicity, while TLR9 gene played no major role in this infection. This study identified the role of TLR3.rs3775290 (c.1377C/T), TLR9.rs5743836 (-1237T-C) and TLR9.rs352140 (G2848A) gene polymorphisms in predicting the outcome of HCV-specific cell-mediated immunity (CMI) among Egyptian healthcare workers (HCWs) and patients. We enrolled 546 subjects (409 HCWs and 137 patients) divided into four groups. Group1: 265 seronegative, aviraemic subjects; group2: 25 seronegative, viraemic subjects; group3: 87 subjects with spontaneously resolved HCV infection; and group4: 169 chronic HCV HCWs and patients. All subjects were genotyped for TLR3.rs3775290, TLR9.rs5743836 and TLR9.rs352140 SNPs by PCR-restriction fragment length polymorphism (PCRRFLP) analysis. We, also, quantified HCV-specific CMI in 265 HCWs distributed among the four groups using an interferon gamma (IFN- $\gamma$ ) enzyme-linked immunospot (ELISpot) assay in response to nine HCV genotype 4a overlapping 15 mer peptide pools covering the whole viral genome. No statistically significant difference was found between CMI responding subjects among Egyptian HCWs with different HCV states and TLR3.rs3775290 genotype or TLR9.rs352140. However, there was a significant relationship between the outcome of the HCV-specific CMI and the TLR9.rs5743836 genotype among the responding subjects $(\mathrm{p}=0.005)$ and the chronic HCV patients $(\mathrm{p}=0.044)$. In conclusion, TLR9.rs5743836 SNP; but not TLR3.rs3775290 or TLR9.rs352140 genotypes; could predict the outcome of HCV-specific CMI responses among genotype-4-infected Egyptians.
\end{abstract}

Association of Toll-like receptor (TLR) 3 \& 9 genes' polymorphism with Hepatitis C virusspecific cell-mediated immunity outcome among Egyptian healthcare workers

Running title : TLR3\&9 SNPs in HCV-infected Egyptians 
Sayed F. Abdelwahab ${ }^{1,2,3, \#,{ }^{*} \text {, Shaimaa Hamdy }}{ }^{1,4, \#}$, Ahmed M. Osman ${ }^{4}$, Zainab A. Zakaria ${ }^{1,5}$, Iman Galal ${ }^{1}$, Maha Sobhy ${ }^{1}$, Mohamed Hashem ${ }^{1,6}$, Walaa R. Allam ${ }^{1,7}$, Mohamed Abdel-Samiee ${ }^{8}$, Eman Rewisha ${ }^{8}$, Imam Waked $^{8}$

${ }^{1}$ The Egyptian Holding Company for Biological Products and Vaccines (VACSERA), 51 Wizaret ElZeraa St., Agouza, Giza 22311, Egypt, ${ }^{2}$ Division of Microbiology, Department of Pharmaceutics and Industrial Pharmacy, Taif College of Pharmacy, Al-Haweiah, PO Box 888, Taif 21974,Kingdom of Saudi Arabia ${ }^{3}$ Department of Microbiology and Immunology, Faculty of Medicine, Minia University, Minia 61511, Egypt, ${ }^{4}$ Department of Zoology, Faculty of Science, Cairo University, Giza 12613, Egypt, ${ }^{5}$ Biomedical Research Laboratory, Faculty of Pharmacy, Heliopolis University for Sustainable Development, Cairo, Egypt, ${ }^{6}$ Department of Epidemiology and Public Health, University of Maryland School of Medicine, Baltimore, MD, USA, ${ }^{7}$ Centre for Genomics, University of Science and Technology, Zewail City of Science and Technology, Giza, Egypt, ${ }^{8}$ Department of Hepatology and Gastroenterology, National Liver Institute, Menoufia University, Menoufia 32511, Egypt

\# Contributed equally to this work

${ }^{*}$ Corresponding Author :

Sayed F. Abdelwahab, PhD

Professor, Department of Microbiology and Immunology, Faculty of Medicine, Minia University, Minia 61511, Egypt and Department of Microbiology, Taif Faculty of Pharmacy, Al-Haweiah, PO Box 888, Taif 21974, KSA

Email: icpminia@yahoo.com

Telephone: +20-10-9000-8885; +966-54-122-1361, Fax: +20-86-234-2813

ORCID: http://orcid.org/0000-0002-9636-7485

Key words: CMI, Cell-mediated immunity; HCV; Healthcare workers; SNP; TLR3; TLR9.

Abbreviations: CMI, Cell-mediated immunity; HCV, hepatitis C virus; HCWs, healthcare workers; TLR, toll-like receptor

Abstract word count: 250; Text word count(Introduction to end of discussion): 3710

\section{Summary:}

Variations in immune responses could define successful resistance to Hepatitis C Virus (HCV) infection. Toll-like receptors (TLR)-3 are innate detectors of dsRNA viruses while TLR9 recognizes bacterial and viral unmethylated CpG motifs. We previously reported that TLR3.rs3775290 "CC" genotype was associated with HCV chronicity, while TLR9 gene played no major role in this infection. This study identified the role of TLR3.rs3775290 (c.1377C/T), TLR9.rs5743836 (-1237T-C) and TLR9.rs352140 (G2848A) gene polymorphisms in predicting the outcome of HCV-specific cell-mediated immunity (CMI) among Egyptian healthcare workers (HCWs) and patients. We enrolled 546 subjects (409 HCWs and 137 patients) divided into four groups. Group1: 265 seronegative, aviraemic subjects; group2: 25 seronegative, viraemic subjects; group3: 87 subjects with spontaneously resolved HCV infection; and group4: 169 chronic HCV HCWs and patients. All subjects were genotyped for TLR3.rs3775290, TLR9.rs5743836 and TLR9.rs352140 SNPs by PCRrestriction fragment length polymorphism (PCR-RFLP) analysis. We, also, quantified HCV-specific CMI in $265 \mathrm{HCWs}$ distributed among the four groups using an interferon gamma (IFN- $\gamma$ ) enzyme-linked immunospot (ELISpot) assay in response to nine HCV genotype 4a overlapping 15mer peptide pools covering the whole viral genome. No statistically significant difference was found between CMI responding subjects among Egyptian HCWs with different HCV states and TLR3.rs3775290 genotype or TLR9.rs352140. However, there was a significant relationship between the outcome of the HCV-specific CMI and the TLR9.rs5743836 genotype among the responding subjects $(p=0.005)$ and the chronic HCV patients $(p=0.044)$. In conclusion, 
TLR9.rs5743836 SNP; but not TLR3.rs3775290 or TLR9.rs352140 genotypes; could predict the outcome of HCV-specific CMI responses among genotype-4-infected Egyptians.

\section{Introduction}

Variations in immune responses may help define successful resistance to Hepatitis C virus (HCV) infection particularly among seronegative healthcare workers, reviewed elsewhere [1]. Toll-like receptors (TLR)-3 are innate detectors of dsRNA of viruses while TLR9 recognizes bacterial and viral unmethylated CpG motifs. $\mathrm{HCV}$ virions bind to the cell surface and enter the cell via receptor-mediated endocytosis. The structure of HCV with different parts are recognized by different TLRs. The core and non-structural (NS) proteins are important sequences recognized by pattern recognition receptors (PRRs), including TLRs. They are, also, important inhibitors of TLR signalling $[2,3]$. HCV core and NS proteins are important pathogen-associated molecular patterns (PAMPs) for TLR2, TLR3, TLR4, TLR7, 8, and 9. TLR3 is important for its antiviral immune effects, and TLR3 stimulated non-parenchymal liver cells are able to regulate HCV replication through production of interferon (IFN) $\beta[4,5]$. TLR3 mRNA is significantly increased in monocytes in chronic HCV infection [6]. An IFN-responsive element has been identified in the promotor region of the TLR3 gene, and it, therefore, seems likely that TLR3 expression is responsive to IFN treatment in HCV infection [7]. Myeloid dendritic cells (mDCs) have normal functioning TLR3 and can produce interleukin (IL)12, IL-6, IL-10, IFN- $\gamma$, and tumour necrosis factor (TNF)- $\alpha$ with TLR3 stimulation despite HCV infection $[8]$.

HCV viral proteins stimulate TLR signalling, which plays an important role in viral immune clearance. However, HCV can simultaneously evade immune clearance through specifically targeting and impairing TLR signalling through several mechanisms. First, HCV interferes with signalling via the TIR-domaincontaining adapter-inducing IFN $\beta$ /TANK-binding kinase (TRIF)/ TBK)1- Interferon regulatory factor 3 (IRF3) pathway. The HCV NS3 protein induces degradation of TRIF, while the NS3/4A protein impedes IRF3 and NF $x \mathrm{~B}$ activation by reducing the amount of TRIF in circulation and by generating cleavage products with dominant-negative activity $[4,9]$. NS3/4A, also, interacts directly with TBK1 to reduce TBK1-IRF3 interaction and, therefore inhibit IRF3 activation [10]. HCV, also, interferes with the TLRMyD88 (Myeloid differentiation primary response 88) pathway through NS5A interaction with MyD88 to prevent Interleukin-1 receptor associated kinase 1 (IRAK1) recruitment and cytokine production in response to ligands for TLR2, TLR4, TLR7, and TLR9 [11].

HCV has been shown to regulate TLR9 expression via transcription factor (Elk-1), which is an important signal integration point between T-Cell Receptor (TCR) and CD28 in T helper 1 (Th1) cell activation [12]. HCV also impairs TLR9-mediated IFN- $\alpha$ and IFN- $\beta$ production and human leukocyte antigen DR (HLADR) expression by pDCs, associated with impaired activation of naïve T cells [13]. TLR9 signalling in mDCs is unaffected $[8,13]$. It is, therefore, clear that compartmentalization of effects on TLR function is a key strategy by which HCV can evade immune clearance yet still lead to chronic inflammatory hepatic damage and liver fibrosis.

We previously showed that TLR3.rs3775290 "CC" genotype was associated with HCV chronicity, while TLR9 gene played no major role in HCV infection [14]. This study identified the role of TLR3.rs3775290 (c.1377C/T), TLR9.rs5743836 (-1237T-C) and TLR9.rs352140 (G2848A) single nucleotide polymorphisms (SNP) in predicting the outcome of HCV-specific cell-mediated immunity (CMI) among Egyptian healthcare workers (HCWs) and patients. We show that TLR9.rs5743836 SNP; but not TLR3.rs3775290 or TLR9.rs352140 genotypes; could predict the outcome of HCV-specific CMI responses among genotype-4infected Egyptians.

\section{Subjects and Methods}

\section{Study subjects}

Two hundred sixty-five HCWs were selected for the evaluation of IFN- $\gamma$ producing T-cells by an Enzyme Linked Immuno-Spot (ELISpot) assay in response to nine HCV genotype 4a overlapping 15mer peptide pools 
covering the whole viral genome. The study protocol was approved by the NLI-Institutional Review Board (IRB), and all subjects gave informed consent prior to enrolment. The demographic characteristics of the participants were previously reported [14].

\section{Laboratory testing}

\section{HCV molecular diagnosis and quantification of HCV-RNA}

HCV diagnosis was determined by using the Murex third generation anti-HCV ELISA assay (version 4.0 USA) that utilizes antigens from the core, NS3, NS4 and NS5 regions of the virus. All the study subjects were tested by quantitative real-time RT-PCR (qPCR) for detection of current HCV infection and quantification of the HCV viral load using QIAamp@ Viral RNA purification kit (QIAGEN, USA). Amplification step was performed using AgPath-ID one-step assay according to the manufacturer's instructions (Applied BiosystemsLife Technologies Corporation, CA).

\section{HCV-genotyping and genotyping for TLR3.rs3775290 (c.1377C/T) SNP}

PCR-RFLP was used for HCV genotyping after reverse transcription as described [15].

PCR-RFLP was also performed to identify TLR3.rs3775290 (c.1377C/T) gene SNP. PCR was carried out in a total reaction volume of $25 \mu \mathrm{l}$ with $20 \mathrm{pmol}$ of each of the forward and reverse primers as described [16].

\section{Genotyping for TLR9.rs5743836 (-1237T-C) and TLR9.rs352140 (G2848A) SNPs}

PCR for TLR9.rs5743836 (-1237T-C) was carried out in a total reaction volume of $25 \mu \mathrm{l}$ with $20 \mathrm{pmol}$ of each of the forward and reverse primers as described [17]. On the other hand, PCR for TLR9.rs352140 (G2848A) was carried out in a total reaction volume of $25 \mu \mathrm{l}$ with $20 \mathrm{pmol}$ of each of the forward and reverse primers as described [16].

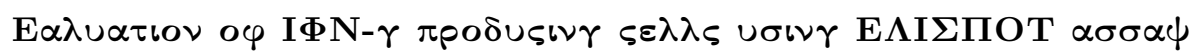

An ELISPOT assay was used to quantify IFN- $\gamma$ production by PBMC isolated from the study subjects in response to nine HCV genotype 4a isolate ED43 peptide antigen pools composed of 15 (15mer) and overlapping by 10 amino acids. These were 600 peptides combined in nine pools corresponding to all the HCV proteins. These synthetic peptides were custom synthesized by Mimotopes (Australia). Approximately $15 \mathrm{ml}$ of whole blood were collected into EDTA vacutainer tubes (Becton Dickinson Biosciences, NJ, USA). PBMC were isolated by Ficoll-Hypaque density gradient centrifugation and viability was determined by trypan blue exclusion method. Briefly, $2 \times 10^{5} \mathrm{PBMC}(200 \mu \mathrm{l} /$ well) were incubated in triplicate cultures in the ELISpot plates (Whatman Unifilter, USA) coated with anti-human IFN $\gamma$ antibody and incubated for 16 hours with or without recombinant HCV antigens at $3 \mu \mathrm{g} / \mathrm{ml}$ of each single peptide in complete RMPI-1640 medium. Negative and positive controls included medium containing DMSO alone and $0.1 \mu \mathrm{g} / \mathrm{ml}$ of SEB or other polyclonal stimuli, respectively. At the end of the incubation period, the assay was developed until the appearance of spots, and then the wells were rinsed with tap water to stop the reaction. The number of spots per well was counted using an automated ELISpot reader (Cellular Technology Ltd., Cleveland, USA) as described [18]. The average number of spot forming cells (SFC) in control wells were subtracted from the average number of peptide-stimulated wells to correct for background cytokine production and are expressed as SFC/million PBMC. A positive HCV antigen-specific response was considered if the SFC in the presence of antigen were at least three-fold the number of SFC in the medium control and if there was $>55 \mathrm{SFC} /$ million PBMC, as we previously reported [19].

\section{Statistical analysis}

Allele and genotype frequencies of each SNP were determined by direct counting. Genotype and allele frequencies between groups were compared using the $\chi^{2}$ test and Fisher's exact test when appropriate. For allelic associations, $p$ values with Yates correction and odds ratio (OR) with 95\% confidence intervals (CI) were calculated using Statcalc program (Epiinfo version 6.0.4, CDC, Atlanta, GA). Pair-wise LD (D', r²) was estimated by use of Haploview software version 4.2. Chi-square tests were performed for categorical 
data, while student's $t$-test (or Mann Whitney U test when appropriate) were performed for comparison of continuous data. Differences in means with a significance value $(\mathrm{p}<0.05)$ were considered significant. Standard errors (and/or deviations) of treatment means were also calculated.

\section{Results}

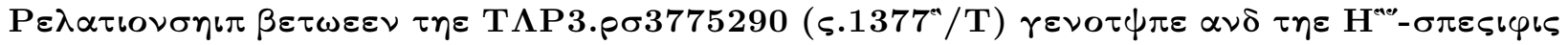

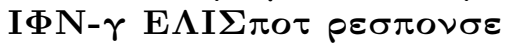

All subjects enrolled into the study were tested for IFN- $\gamma$ production by PBMCs using ELISpot assay to evaluate CMI response against nine HCV genotype 4a isolate ED43 peptide antigen pools composed of 15 (15mer) and overlapping by 10 amino acids. Only 140 (53\%), 20 (80\%), 35 (40\%) and $70(41.4 \%)$ samples had valid CMI data among the four study groups (seronegative, aviraemic subjects; seronegative, viraemicsubjects; spontaneously resolved subjects and chronic HCV patients groups, respectively).

There was no relationship between the outcome of the HCV-specific immune response and the TLR3.rs3775290 genotype among the $263 \mathrm{HCWs}$ with valid CMI responses and TLR3.rs3775290 genotyping tests $(p=0.222)$. Also, there was a significantly $(p=0.004)$ higher proportion of the CC genotype $(73.3 \%)$ in responding subjects than in non-responding seronegative, aviraemic subjects (65.1\%). In addition, there was a differential distribution of the three TLR3.rs3775290 genotypes among the total subjects with and without HCV-specific CMI (Table 1).

The average totals $( \pm \mathrm{SEM})$ of IFN- $\gamma$ responses measured in SFC per million PBMCs in the responding total subjects were $455 \pm 90,507 \pm 122$, and $317 \pm 45$ with the TLR3.rs3775290 genotype CC $(\mathrm{n}=37), \mathrm{CT}(\mathrm{n}=16)$ and $\mathrm{TT}(\mathrm{n}=6)$. On the other hand, among the non-responding subjects, the average totals ( \pm SEM) of IFN- $\gamma$ SFC per million PBMCs in total subjects were $29 \pm 5,23 \pm 5$, and $49 \pm 19$ with the TLR3.rs3775290 genotype CC $(\mathrm{n}=130), \mathrm{CT}(\mathrm{n}=65)$ and TT $(\mathrm{n}=9)$, respectively. There was no significant difference between the responding total subjects with different TLR3.rs3775290 genotypes ( $p=0.89$; Figure 1A).

For the seronegative, aviraemic subjects, the average totals of HCV-specific IFN- $\gamma$ SFC $/ 10^{6} \mathrm{PBMCs}( \pm \mathrm{SEM})$ of the responding subjects were $404 \pm 97.7,1058 \pm 807.5$, and $249 \pm 21.9$, among those with the TLR3.rs3775290 CC genotype $(\mathrm{n}=22)$, CT $(\mathrm{n}=4)$ and TT $(\mathrm{n}=4)$ genotypes, respectively. Differences were not statistically significant $(p=0.88)$. On the other hand, the average totals of IFN $-\gamma$ SFC $/ 10^{6}$ PBMCs $( \pm$ SEM) of the non-responding subjects were $26.7 \pm 5.7,16.7 \pm 4$, and $13.3 \pm 13.3$, among those with the TLR3.rs3775290 CC $(\mathrm{n}=71)$, CT $(\mathrm{n}=36)$ and TT $(\mathrm{n}=2)$ genotypes, respectively (Figure $1 \mathrm{~B})$.

Regarding seronegative, viraemic subjects, the average totals ( $\pm \mathrm{SEM})$ of $\mathrm{HCV}$-specific IFN- $\gamma \mathrm{SFC} / 10^{6}$ PBMCs forresponding subjects were $657 \pm 443$, and 163, among those with the TLR3.rs3775290 genotype $\mathrm{CC}(\mathrm{n}=3)$ and $\mathrm{CT}(\mathrm{n}=1)$ genotypes, respectively. There were no responding subjects with the TT genotype. Differences were not statistically significant $(p=0.18)$. On the other hand, the average totals of IFN- $\gamma$ SFC $/ 10^{6}$ PBMCs ( \pm SEM) of the non-responding subjects were $42 \pm 22,30 \pm 11$ and $8 \pm 8$, among those with the TLR3.rs3775290 CC (n=9), CT $(\mathrm{n}=5)$ and TT $(\mathrm{n}=2)$ genotypes, respectively (Figure 1C).

For spontaneously resolved subjects, the average totals of HCV-specific IFN- $\gamma$ SFC $/ 10^{6}$ PBMCs ( \pm SEM) of theresponding subjects were $793 \pm 605.8$, and $325 \pm 121$, among those with the TLR3.rs3775290 CC ( $\mathrm{n}=2)$, CT $(n=5)$ genotypes, respectively. No TT genotype was recorded in this category. Differences were not statistically significant $(p=0.698)$. On the other hand, the average totals of IFN- $\gamma$ SFC $/ 10^{6} \mathrm{PBMCs}( \pm \mathrm{SEM})$ of the non-responding subjects were $51 \pm 21,15 \pm 12$ and 58 , among those with the TLR3.rs $3775290 \mathrm{CC}(\mathrm{n}=14)$, CT $(\mathrm{n}=12)$ and TT $(\mathrm{n}=1)$ genotypes, respectively (Figure 1D).

For chronic HCV patients, the average totals of HCV-specific IFN- $\gamma$ SFC $/ 10^{6}$ PBMCs $( \pm \mathrm{SEM})$ of the respondingsubjects were $439 \pm 214,349 \pm 181$, and $453 \pm 9$, among those with the TLR3.rs3775290 CC (n=10), CT $(n=6)$ and TT $(n=2)$ genotypes, respectively. Differences were not statistically significant $(p=0.228)$. On the other hand, the average totals of IFN $-\gamma$ SFC $/ 10^{6}$ PBMCs $( \pm$ SEM) of the non-responding chronic HCV patients were $26 \pm 8,38 \pm 13$, and $84 \pm 36$, among those with the TLR3.rs3775290 CC ( $\mathrm{n}=36)$, CT $(\mathrm{n}=13)$ and TT $(\mathrm{n}=4)$ genotypes, respectively (Figure $1 \mathrm{E})$. 


\section{Relationship between the TLR9.rs5743836 (-1237T-C) genotype and the HCV-specific IFN ELISpot response}

There was no relationship between the outcome of the HCV-specific CMI response and the TLR9.rs5743836 genotype among the $265 \mathrm{HCWs}$ with valid CMI responses and TLR9.rs5743836 genotyping tests (Table $2, p=0.12)$. However, there was a statistically significant $(p=0.019)$ lower proportion of the TT genotype in responding spontaneously resolved subjects compared to non-responding spontaneously resolved subjects (20\% Vs $80 \%$, respectively; Table 2).

The average totals $( \pm \mathrm{SEM})$ of IFN $-\gamma$ responses measured in SFC per million PBMCs in the responding total subjects were $624 \pm 125$, and $381 \pm 100$ with the TLR9.rs5743836 CT $(\mathrm{n}=18)$ and TT $(\mathrm{n}=41)$ genotypes, respectively. There were no responding subjects with the CC genotype. On the other hand, among the non-responding subjects, the average totals $( \pm \mathrm{SEM})$ of IFN- $\gamma$ SFC per million PBMCs in total subjects

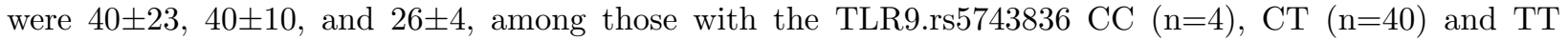
$(\mathrm{n}=162)$, respectively. There was a significant difference between the responding total subjects with different TLR9.rs5743836 genotypes ( $p=0.005$; Figure 2A).

For the seronegative, aviraemic subjects, the average totals of HCV-specific IFN- $\gamma \mathrm{SFC} / 10^{6} \mathrm{PBMCs}( \pm \mathrm{SEM})$ of theresponding subjects were $617 \pm 192$, and $398 \pm 164$, among those with the TLR9.rs5743836 CT ( $\mathrm{n}=10)$ and TT $(n=20)$ genotypes, respectively. There were no responding subjects with the CC genotype. Differences were not statistically significant $(p=0.159)$. On the other hand, the average totals of IFN- $\gamma \mathrm{SFC} / 10^{6} \mathrm{PBMCs}$ ( \pm SEM) of the non-responding seronegative, aviraemic subjects were $32 \pm 31,44 \pm 14$, and $18 \pm 3$, among those with the TLR9.rs5743836 CC ( $\mathrm{n}=3)$, CT $(\mathrm{n}=25)$ and TT $(\mathrm{n}=82)$ genotypes, respectively (Figure 2B).

For seronegative, viraemic subjects, the average totals of $\mathrm{HCV}$-specific IFN $-\gamma \mathrm{SFC} / 10^{6} \mathrm{PBMCs}( \pm \mathrm{SEM})$ of theresponding subjects were 238, and 632 \pm 455 , among those with the TLR9.rs5743836 CT $(\mathrm{n}=1)$ and TT $(\mathrm{n}=3)$ genotypes, respectively. There were no responding subjects with the $\mathrm{CC}$ genotype. Differences were not statistically significant $(p=0.654)$. On the other hand, the average totals of IFN- $\gamma \mathrm{SFC} / 10^{6} \mathrm{PBMCs}$ ( \pm SEM) of the non-responding subjects were $65,18 \pm 14$, and $38 \pm 18$, among those with the TLR9.rs5743836 $\mathrm{CC}(\mathrm{n}=1), \mathrm{CT}(\mathrm{n}=4)$ and TT $(\mathrm{n}=11)$ genotypes, respectively (Figure $2 \mathrm{C})$.

For spontaneously resolved subjects, the average total HCV-specific IFN- $\gamma \mathrm{SFC} / 10^{6} \mathrm{PBMCs}( \pm)$ of theresponding subjects was $795 \pm 345$, and $206 \pm 49$, among those with the TLR9.rs5743836 CT $(\mathrm{n}=3)$ and TT $(\mathrm{n}=4)$ genotypes, respectively. There were no responding subjects with the $\mathrm{CC}$ genotype. Differences were not statistically significant $(p=0.077)$. On the other hand, the average totals of IFN- $\gamma$ SFC $/ 10^{6} \mathrm{PBMCs}( \pm$ ) ofnon-responding subjects were 1 , and 36 \pm 12 , among those with the TLR9.rs5743836 CT (n=1) and TT $(\mathrm{n}=27)$ genotypes, respectively. Also, there were no subjects with the $\mathrm{CC}$ genotype among non-responding subjects (Figure 2D).

For chronic HCV patients, the average totals of HCV-specific IFN- $\gamma$ SFC/10 ${ }^{6}$ PBMCs $( \pm$ SEM) of the respondingsubjects were $610 \pm 212$, and $354 \pm 156$, among those with the TLR9.rs5743836, CT $(\mathrm{n}=4)$ and TT $(\mathrm{n}=14)$ genotypes, respectively. There were no responding subjects with the CC genotype. Differences were statistically significant $(p=0.044)$. On the other hand, the average totals of IFN- $\gamma$ SFC $/ 10^{6} \mathrm{PBMCs}( \pm \mathrm{SEM})$ of the non-responding chronic HCV subjects were $43 \pm 19$ and $32 \pm 8$, among those with the TLR9.rs5743836 CT $(n=10)$ and TT $(n=42)$ genotypes, respectively. There were no subjects with the CC genotype among the non-responding chronic patients (Figure 2E).

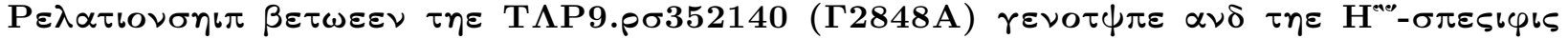

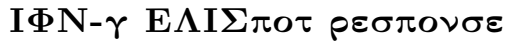

There was no relationship between the outcome of the HCV-specific CMI response and the TLR9.rs352140 (G2848A) genotype among the 258 HCWs subjects with valid CMI responses and TLR9.rs352140 genotyping tests $(p=0.356)$. Also, there was no significant difference $(p=0.299)$ in the proportion of the GG genotype in responding (46.4\%) and in the non-responding seronegative aviraemic subjects (31.4\%; Table 3).

The average totals $( \pm \mathrm{SEM})$ of IFN- $\gamma$ responses measured in SFC per million PBMCs in the responding total 
subjects were $587 \pm 176,390 \pm 85$, and $327 \pm 45$, among those with the TLR9.rs352140 GG ( $\mathrm{n}=24)$, GA ( $\mathrm{n}=23$ ) and AA $(n=10)$ genotypes, respectively. On the other hand, among the non-responding subjects, the average totals $( \pm$ SEM) of IFN- $\gamma$ SFC per million PBMCs in the total subjects were $30 \pm 6,31 \pm 6$, and $26 \pm 7$, among those with the TLR9.rs352140 (G2848A) GG $(n=66)$, GA $(n=86)$ and AA $(n=49)$ genotypes, respectively. There was no significant difference between the responders of the total subjects with the TLR9.rs352140 genotypes $(p=0.652$; Figure $3 \mathrm{~A})$.

For the seronegative, aviraemic subjects, the average totals of HCV-specific IFN- $\gamma$ SFC $/ 10^{6}$ PBMCs $( \pm$ SEM) of theresponding subjects was $672 \pm 278.8,307 \pm 86$, and $357 \pm 68$, among those with the TLR9.rs352140 GG $(\mathrm{n}=13), \mathrm{GA}(\mathrm{n}=10)$ and AA $(\mathrm{n}=5)$ genotypes, respectively. Differences were not statistically significant $(p$ $=0.594)$. On the other hand, the average totals of IFN $\gamma$ SFC $/ 10^{6} \mathrm{PBMCs}( \pm \mathrm{SEM})$ among the non-responding seronegative, aviraemic subjects were $23 \pm 7,31 \pm 8$, and $17 \pm 5$, among those with the TLR9.rs352140 GG $(\mathrm{n}=33)$, GA $(\mathrm{n}=43)$ and AA $(\mathrm{n}=29)$ genotypes, respectively (Figure 3B).

For seronegative, viraemic subjects, the average totals of HCV-specific IFN- $\gamma$ SFC $/ 10^{6}$ PBMCs $( \pm$ SEM) of the responding subjects were $214 \pm 24.2$, and $853 \pm 689$, among those with the TLR9.rs352140 GG $(\mathrm{n}=2)$ and GA $(n=2)$ genotypes, respectively. There were no responding subjects with the AA genotype. Differences were not statistically significant $(p=1.000)$. On the other hand, the average totals of IFN- $\gamma$ SFC $/ 10^{6} \mathrm{PBMCs}$ ( \pm SEM) were $12 \pm 2.22,30 \pm 10.4$, and $60 \pm 47.6$, among the non-responding seronegative, viraemic subjects with the TLR9.rs352140 (G2848A) GG $(\mathrm{n}=3)$, GA $(\mathrm{n}=9)$ and AA $(\mathrm{n}=4)$ genotypes, respectively (Figure $3 \mathrm{C})$.

For spontaneously resolved subjects, the average totals of HCV-specific IFN- $\gamma$ SFC $/ 10^{6}$ PBMCs $( \pm$ SEM) of theresponding subjects were $244 \pm 48.2$, and $619 \pm 301$, among those with the TLR9.rs352140 GG ( $\mathrm{n}=3)$ and GA $(n=4)$ genotypes, respectively. There were no responding subjects with the AA genotype. Differences were not statistically significant $(p=1.000)$. On the other hand, the average totals of IFN- $\gamma$ SFC $/ 10^{6}$ PBMCs ( \pm SEM) were $26 \pm 14.1,60 \pm 26.9$, and $10 \pm 10$, among the non-responding spontaneously resolved subjects with the TLR9.rs352140 GG ( $\mathrm{n}=11)$, GA $(\mathrm{n}=10)$ and AA $(\mathrm{n}=7)$ genotypes, respectively (Figure 3D).

For chronic HCV patients, the average totals of HCV-specific IFN- $\gamma$ SFC $/ 10^{6}$ PBMCs $( \pm \mathrm{SEM}$ ) of the responding subjects were $698 \pm 357.2,245 \pm 51.5$, and $296 \pm 57.8$, among those with the TLR9.rs352140 GG $(\mathrm{n}=6)$, GA $(\mathrm{n}=7)$ and AA $(\mathrm{n}=5)$ genotypes, respectively. Differences were not statistically significant $(p$ $=0.775$ ). On the other hand, the average totals of IFN $-\gamma \mathrm{SFC} / 10^{6} \mathrm{PBMCs}( \pm \mathrm{SEM})$ of non-responding

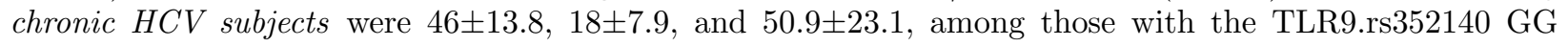
$(\mathrm{n}=19)$, GA $(\mathrm{n}=24)$ and AA $(\mathrm{n}=9)$ genotypes, respectively (Figure $3 \mathrm{E})$.

\section{Discussion}

In this study, there was no statistically significant difference between CMI responding subjects in Egyptian HCWs with different HCV states with different TLR3.rs3775290 (c.1377C/T) genotypes. Also, there was no relationship between the outcome of the HCV-specific CMI response and the TLR3.rs3775290 (c.1377C/T) genotype among the 263 subjects tested. This agrees with another report [20]. The role of TLR3 was investigated in four different infectious viral models including lymphocytic choriomeningitis virus (LCMV), vesicular stomatitis virus (VSV), Murine cytomegalovirus (MCMV), and reovirus in TLR3 ${ }^{-/-}$mice. The investigators found that TLR3 is not always required for the generation of effective antiviral responses, as the absence of TLR3 did not alter either viral pathogenesis or host's generation of adaptive antiviral responses to those viruses. Interestingly, intracellular transduction of poly I:C initiates activation of an IFN response in a TLR3-independent manner, thus limiting the role of TLR3 in the IFN pathway [21]. Other reports [22, 23] indicate that HCV may use TLR3 pathway to evade immune surveillance via HCV NS3/4A proteasemediated cleavage of the TLR3 adaptor protein TRIF. Also, HCV NS3/4A interferes with RIG-I, a key factor in TRIF-independent signalling [21]. In addition, it was suggested that several polymorphisms that alter TLR3 amino acids initiate resultant changes in the protein and that they might downregulate the gene expression and lower the activities of TLR3 required for proper signalling [24]. Reduced activity of TLR3 results in failure to recognize invading microorganisms and insufficient immune responses, thus increasing 
the likelihood of infections and infectious diseases [25].

Our data provide strong indirect evidence that TLR9 might play a greater role in HCV infection than previously expected. We identified an association of the polymorphism TLR9.rs5743836 within the TLR9 gene with the natural course of HCV infection. In this study, there was a strong relationship between the outcome of the HCV-specific CMI response and the TLR9.rs5743836 (-1237T-C) genotype among the 265 subjects with valid CMI responses in responders of total subjects of HCWs $(p=0.005)$. Also, there was a statistically significant difference between responding subjects in chronic HCV patients with different TLR9.rs5743836 $(p=0.044)$.

We show that the TLR9-1237T allele was significantly associated with HCV-specific CMI response. In this regard, the TT genotype is transcribed more effectively than the CC genotype as reported by others [26] who showed that the wild-type construct elicits higher transcriptional activity compared to the variant CC allele. Data showed that subjects with the "favourable" TT allele would have HCV-specific CMI response when compared to those with the "unfavourable" CC allele. Also, several reports found that the mutant allele "T" imparts the immunity against the various pathogens. However, statistically inconsiderable, the mutant allele was declared to be linked with the depressed microbial load in Africans [27-29]. In addition, the mutant allele T manipulate immunity against establishing the infections, which is convincing that the TLR9 gene had bear the influence of genetic assortment to cope with the infections [30]. In contrast, functional analyses of the impact of the TLR9 polymorphisms on basal promoter activity revealed that the rs 5743836 SNP provoke higher gene expression compared with the wild type promoter. Increased promoter activity of TLR9.rs573836 might be explained by the findings of others [31], who showed that the variant C allele at rs5743836 creates a potential NF- $\varkappa$ B-binding site that increased the transcriptional activity of the gene. The presence of this extra putative NF- $x \mathrm{~B}$-binding site promotes TLR 9 transcription in response to various stimuli more effectively than the wild type TLR9.rs5743836T sequence. This finding agrees with another report [32], TLR9 promoter SNPs are associated with the natural course of HCV infection and show higher transcriptional activities and imply the DNA sensor TLR9 in natural immunity against the RNA virus, HCV. Our data are analogous to another study $[1,33]$, where the rs5743836 TT variant has been shown to have a higher promoter activity than the CC genotype. That study suggested that this activity could result in an increased pro-inflammatory cytokine production during malaria infection leading to successful control and elimination of malaria parasites [33].

There was no relationship between the outcome of the HCV-specific immune response and the TLR9.rs352140 (G2848A) genotype among the 258 Egyptian HCWs subjects with valid CMI responses and TLR9.rs352140 genotyping. To date, there have been no reports showing an association between TLR9.rs352140 (G2848A) and HCV infection. The region around 2848 is the major coding region of the TLR9 protein [34, 35] and a polymorphism of the TLR9 2848 GA genotype has been reported to reduce TLR9 expression at the transcriptional level $[35,36]$. Down regulated TLR9 could reduce the functions of the innate immune reactivity against HCV infection. Our data are analogous to another study which suggest that the TLR9 gene may play a role in cervical carcinogenesis but have a lesser (or no) role in tumour progression where subjects with the favourable" GG allele were reported to be more frequent than the "unfavourable" AA allele [37]. In addition, a recent meta-analysis demonstrated that there is no association between TLR9.rs352140 (G2848A) and cervical cancer susceptibility [38]. Our findings suggest that the TLR9.rs352140 (G2848A) genotype does not affect the natural course of HCV infection.

In conclusion, this study shows that TLR9.rs5743836 SNP; but not TLR3.rs3775290 or TLR9.rs352140 genotypes; could predict the outcome of $\mathrm{HCV}$-specific CMI responses among genotype-4-infected Egyptians.

Acknowledgement : This study was supported by the Egyptian Science and Technology Development Fund (STDF) Contract no. 1664 to S.F. Abdelwahab.

\section{Author contributions :}

Conceptualization and design of experiments: SFA, SH, AO, ZZ, ER, and IW; Data collection, experimentation, analysis, and investigation: SH, SFA, MA, ZZ, IG, MS, WA; Funding acquisition and resources: SFA, 
ER, and IW; Writing-original draft (SH and SFA); Writing-review and editing (SFA, AO, MS, MH, WA, MA, ER, and IW). All authors approved the final version of the manuscript.

Compliance with Ethical Standards :

Conflict of Interest: None declared.

Data Sharing and Data Accessibility: All data pertinent to this article are included herein.

Research involving human participants and/or animals :

All procedures performed in studies involving human participants were in accordance with the ethical standards of the institutional and/or national research committee and with the 1964 Helsinki declaration and its later amendments.

Informed Consent : Informed consent was obtained from all enrolled study participants.

Table (1): Relationship between TLR3.rs3775290 (c.1377C/T) and HCV-specific cell mediated immune (CMI) response among NLI HCWs in terms of CMI responders frequencies.

\begin{tabular}{|c|c|c|c|c|}
\hline$p$-value & Total & TLR3.rs3775290 genotype & TLR3.rs3775290 genotype & TLR3.rs3775290 genotype \\
\hline & & TT & CT & $\mathrm{CC}$ \\
\hline \multirow[t]{2}{*}{0.004} & $30(21.6)$ & $4(13.3)$ & $4(13.3)$ & $22(73.3)$ \\
\hline & $109(78.4)$ & $2(1.8)$ & $36(33)$ & $71(65.1)$ \\
\hline \multirow[t]{2}{*}{0.694} & $4(20)$ & $0(0)$ & $1(25)$ & $3(75)$ \\
\hline & $16(80)$ & $2(12.5)$ & $5(31.3)$ & $9(56.3)$ \\
\hline \multirow[t]{2}{*}{0.425} & $7(20.6)$ & $0(0)$ & $5(71.4)$ & $2(28.6)$ \\
\hline & $27(79.4)$ & $1(3.7)$ & $12(44.4)$ & $14(51.9)$ \\
\hline \multirow[t]{2}{*}{0.57} & $18(25.7)$ & $2(11.1)$ & $6(33.3)$ & $10(55.5)$ \\
\hline & $52(74.3)$ & $4(7.7)$ & $12(23.1)$ & $36(69.2)$ \\
\hline \multirow[t]{2}{*}{0.222} & $59(22.3)$ & $6(10.2)$ & $16(27.1)$ & $37(62.7)$ \\
\hline & $204(77.7)$ & $9(4.4)$ & $65(31.9)$ & $130(63.7)$ \\
\hline
\end{tabular}

Table (2) : Relationship between TLR9.rs5743836 (-1237T-C) and HCV-specific cell mediated immune (CMI) response among NLI HCWs in terms of CMI responders frequencies.

\begin{tabular}{|c|c|c|c|c|}
\hline$p$-value & Total & TLR9.rs5743836 genotype & TLR9.rs5743836 genotype & TLR9.rs5743836 genotype \\
\hline & & $\mathbf{T T}$ & CT & $\mathrm{CC}$ \\
\hline \multirow{2}{*}{0.353} & $30(21.4)$ & $20(66.7)$ & $10(33.3)$ & $0(0)$ \\
\hline & $110(78.5)$ & $82(74.5)$ & $25(22.7)$ & $3(2.7)$ \\
\hline \multirow[t]{2}{*}{0.87} & $4(20)$ & $3(75)$ & $1(25)$ & $0(0)$ \\
\hline & $16(80)$ & $11(68.8)$ & $4(25)$ & $1(6.3)$ \\
\hline \multirow[t]{2}{*}{0.019} & $7(20)$ & $4(57.1)$ & $3(42.9)$ & $0(0)$ \\
\hline & $28(80)$ & $27(96.4)$ & $1(3.6)$ & $0(0)$ \\
\hline \multirow[t]{2}{*}{0.74} & $18(25.7)$ & $14(77.8)$ & $4(22.2)$ & $0(0)$ \\
\hline & $52(74.3)$ & $42(80.8)$ & $10(19.2)$ & $0(0)$ \\
\hline \multirow[t]{2}{*}{0.12} & $59(22.3)$ & $41(69.5)$ & $18(30.5)$ & $0(0)$ \\
\hline & $206(77.7)$ & $162(78.6)$ & $40(19.4)$ & $4(1.9)$ \\
\hline
\end{tabular}

Table (3) : Relationship between TLR9.rs352140 (G2848A) and HCV-specific cell mediated immune (CMI) response among NLI HCWs in terms of CMI responders frequencies. 


\begin{tabular}{|c|c|c|c|c|c|}
\hline$p$-value & Total & TLR9.rs352140 genotype & TLR9.rs352140 genotype & TLR9.rs352140 genotype & GRC \\
\hline & & AA & GA & GG & \\
\hline \multirow[t]{2}{*}{0.299} & $28(21.1)$ & $5(17.9)$ & $10(35.7)$ & $13(46.4)$ & Res \\
\hline & $105(78.9)$ & $29(27.6)$ & $43(41)$ & $33(31.4)$ & Nol \\
\hline \multirow[t]{2}{*}{0.32} & $4(20)$ & $0(0)$ & $2(50)$ & $2(50)$ & Res \\
\hline & $16(80)$ & $4(25)$ & $9(56.3)$ & $3(18.8)$ & No \\
\hline \multirow[t]{2}{*}{0.299} & $7(20)$ & $0(0)$ & $4(57.1)$ & $3(42.9)$ & $\operatorname{Res}$ \\
\hline & $28(80)$ & $7(25)$ & $10(35.7)$ & $11(39.3)$ & No \\
\hline \multirow[t]{2}{*}{0.627} & $18(25.7)$ & $5(27.8)$ & $7(38.9)$ & $6(33.3)$ & Res \\
\hline & $52(74.3)$ & $9(17.3)$ & $24(46.2)$ & $19(36.5)$ & No \\
\hline \multirow[t]{2}{*}{0.35} & $57(22.1)$ & $10(17.5)$ & $23(40.4)$ & $24(42.1)$ & $\mathbf{R e}$ \\
\hline & $201(77.9)$ & $49(24.4)$ & $86(42.8)$ & $66(32.8)$ & No \\
\hline
\end{tabular}

\section{References}

1. Abdelwahab SF. Cellular immune response to hepatitis-C-virus in subjects without viremia or seroconversion: is it important? Infectious agents and cancer 2016; $11: 23$.

2. Ghany MG, Strader DB, Thomas DL, Seeff LB, American Association for the Study of Liver D. Diagnosis, management, and treatment of hepatitis C: an update. Hepatology 2009; 49 :1335-74.

3. Rosen HR. Clinical practice. Chronic hepatitis C infection. N Engl J Med 2011; 364 :2429-38.

4. Li K, Chen Z, Kato N, Gale M, Jr., Lemon SM. Distinct poly(I-C) and virus-activated signaling pathways leading to interferon-beta production in hepatocytes. J Biol Chem 2005; 280 :16739-47.

5. Broering R, Wu J, Meng Z, Hilgard P, Lu M, Trippler M, Szczeponek A, Gerken G, Schlaak JF. Toll-like receptor-stimulated non-parenchymal liver cells can regulate hepatitis C virus replication. J Hepatol 2008; $48: 914-22$.

6. Dolganiuc A, Garcia C, Kodys K, Szabo G. Distinct Toll-like receptor expression in monocytes and T cells in chronic HCV infection.World J Gastroenterol 2006; 12 :1198-204.

7. Geiss GK, Carter VS, He Y, Kwieciszewski BK, Holzman T, Korth MJ, Lazaro CA, Fausto N, Bumgarner RE, Katze MG. Gene expression profiling of the cellular transcriptional network regulated by alpha/beta interferon and its partial attenuation by the hepatitis $\mathrm{C}$ virus nonstructural 5A protein. J Virol 2003; 77 :6367-75.

8. Shiina M, Rehermann B. Cell culture-produced hepatitis C virus impairs plasmacytoid dendritic cell function. Hepatology 2008;47 :385-95.

9. Foy E, Li K, Wang C, Sumpter R, Jr., Ikeda M, Lemon SM, Gale M, Jr. Regulation of interferon regulatory factor-3 by the hepatitis $\mathrm{C}$ virus serine protease. Science $2003 ; \mathbf{3 0 0}: 1145-8$.

10. Otsuka M, Kato N, Moriyama M, Taniguchi H, Wang Y, Dharel N, Kawabe T, Omata M. Interaction between the HCV NS3 protein and the host TBK1 protein leads to inhibition of cellular antiviral responses.Hepatology 2005; 41 :1004-12.

11. Abe T, Kaname Y, Hamamoto I, Tsuda Y, Wen X, Taguwa S, Moriishi K, Takeuchi O, Kawai T, Kanto T, Hayashi N, Akira S, Matsuura Y. Hepatitis C virus nonstructural protein 5A modulates the toll-like receptor-MyD88-dependent signaling pathway in macrophage cell lines. J Virol 2007; 81 :8953-66.

12. Fukuda K, Tsuchihara K, Hijikata M, Nishiguchi S, Kuroki T, Shimotohno K. Hepatitis C virus core protein enhances the activation of the transcription factor, Elk1, in response to mitogenic stimuli.Hepatology 2001; 33 :159-65. 
13. Yonkers NL, Rodriguez B, Milkovich KA, Asaad R, Lederman MM, Heeger PS, Anthony DD. TLR ligand-dependent activation of naive CD4 T cells by plasmacytoid dendritic cells is impaired in hepatitis C virus infection. J Immunol 2007; $178: 4436-44$.

14. Hamdy S, Osman AM, Zakaria ZA, Galal I, Sobhy M, Hashem M, Allam WR, Abdel-Samiee M, Rewisha E, Waked I, Abdelwahab SF. Association of Toll-like receptor 3 and Toll-like receptor 9 single-nucleotide polymorphisms with hepatitis C virus persistence among Egyptians.Arch Virol 2018; 163 :2433-42.

15. Davidson F, Simmonds P. Determination of HCV Genotypes by RFLP. Methods Mol Med 1999; 19 :175-81.

16. Pandey S, Mittal B, Srivastava M, Singh S, Srivastava K, Lal P, Mittal RD. Evaluation of Toll-like receptors 3 (c.1377C/T) and 9 (G2848A) gene polymorphisms in cervical cancer susceptibility.Mol Biol Rep $2011 ; 38: 4715-21$.

17. Demirci FY, Manzi S, Ramsey-Goldman R, Kenney M, Shaw PS, Dunlop-Thomas CM, Kao AH, Rhew EY, Bontempo F, Kammerer C, Kamboh MI. Association study of Toll-like receptor 5 (TLR5) and Toll-like receptor 9 (TLR9) polymorphisms in systemic lupus erythematosus. J Rheumatol 2007; 34 :1708-11.

18. Abdelwahab SF, Zakaria Z, Sobhy M, Rewisha E, Mahmoud MA, Amer MA, Del Sorbo M, Capone S, Nicosia A, Folgori A, Hashem M, El-Kamary SS. Hepatitis C virus-multispecific T-cell responses without viremia or seroconversion among Egyptian health care workers at high risk of infection. Clin Vaccine Immunol 2012; $19: 780-6$.

19. Farid A, Al-Sherbiny M, Osman A, Mohamed N, Saad A, Shata MT, Lee DH, Prince AM, Strickland GT. Schistosoma infection inhibits cellular immune responses to core HCV peptides. Parasite Immunol 2005;27 :189-96.

20. Edelmann KH, Richardson-Burns S, Alexopoulou L, Tyler KL, Flavell RA, Oldstone MB. Does Toll-like receptor 3 play a biological role in virus infections? Virology 2004; 322 :231-8.

21. Takeuchi O, Hemmi H, Akira S. Interferon response induced by Toll-like receptor signaling. J Endotoxin Res 2004;10 :252-6.

22. Li K, Foy E, Ferreon JC, Nakamura M, Ferreon AC, Ikeda M, Ray SC, Gale M, Jr., Lemon SM. Immune evasion by hepatitis $\mathrm{C}$ virus NS3/4A protease-mediated cleavage of the Toll-like receptor 3 adaptor protein TRIF. Proc Natl Acad Sci U S A 2005; 102 :2992-7.

23. Chang S, Kodys K, Szabo G. Impaired expression and function of toll-like receptor 7 in hepatitis C virus infection in human hepatoma cells. Hepatology 2010; $51: 35-42$.

24. Ranjith-Kumar CT, Miller W, Sun J, Xiong J, Santos J, Yarbrough I, Lamb RJ, Mills J, Duffy KE, Hoose S, Cunningham M, Holzenburg A, Mbow ML, Sarisky RT, Kao CC. Effects of single nucleotide polymorphisms on Toll-like receptor 3 activity and expression in cultured cells. J Biol Chem 2007; 282 :17696-705.

25. Geng PL, Song LX, An H, Huang JY, Li S, Zeng XT. Toll-Like Receptor 3 is Associated With the Risk of HCV Infection and HBV-Related Diseases. Medicine (Baltimore) 2016; 95 :e2302.

26. Novak N, Yu CF, Bussmann C, Maintz L, Peng WM, Hart J, Hagemann T, Diaz-Lacava A, Baurecht HJ, Klopp N, Wagenpfeil S, Behrendt H, Bieber T, Ring J, Illig T, Weidinger S. Putative association of a TLR9 promoter polymorphism with atopic eczema. Allergy 2007;62 :766-72.

27. Christensen SR, Kashgarian M, Alexopoulou L, Flavell RA, Akira S, Shlomchik MJ. Toll-like receptor 9 controls anti-DNA autoantibody production in murine lupus. J Exp Med 2005; 202 :321-31.

28. Yusuf JH, Kaliyaperumal D, Jayaraman M, Ramanathan G, Devaraju P. Genetic selection pressure in TLR9 gene may enforce risk for SLE in Indian Tamils. Lupus 2017; 26 :307-10. 
29. Medhi S, Deka M, Deka P, Swargiary SS, Hazam RK, Sharma MP, Gumma PK, Asim M, Kar P. Promoter region polymorphism \& expression profile of toll like receptor-3 (TLR-3) gene in chronic hepatitis $\mathrm{C}$ virus (HCV) patients from India. Indian J Med Res 2011; 134 :200-7.

30. Wei XS, Wei CD, Tong YQ, Zhu CL, Zhang PA. Single nucleotide polymorphisms of toll-like receptor 7 and toll-like receptor 9 in hepatitis C virus infection patients from central China. Yonsei Med J 2014; 55 :428-34.

31. Ng MT, Van't Hof R, Crockett JC, Hope ME, Berry S, Thomson J, McLean MH, McColl KE, El-Omar EM, Hold GL. Increase in NF-kappaB binding affinity of the variant $\mathrm{C}$ allele of the toll-like receptor 9 1237T/C polymorphism is associated with Helicobacter pylori-induced gastric disease. Infect Immun 2010; $78: 1345-52$.

32. Fischer J, Weber AN, Bohm S, Dickhofer S, El Maadidi S, Deichsel D, Knop V, Klinker H, Moller B, Rasenack J, Wang L, Sharma M, Hinrichsen H, Spengler U, Buggisch P, Sarrazin C, Pawlita M, Waterboer T, Wiese M, Probst-Muller E, Malinverni R, Bochud PY, Gardiner CM, O'Farrelly C, Berg T. Sex-specific effects of TLR9 promoter variants on spontaneous clearance of HCV infection. Gut 2016.

33. Aslam N, Batool F, Iqbal MS, Ashfaq UA, Ayaz S, Khaliq S, Jahan S, Ghani MU, Usman M. Analysis of toll-like receptors-9 (TLR9) gene polymorphism (rs5743836) in Pakistani patients with HCV. Pak J Pharm Sci 2018; $31: 2709-14$.

34. Lange NE, Zhou X, Lasky-Su J, Himes BE, Lazarus R, Soto-Quiros M, Avila L, Celedon JC, Hawrylowicz CM, Raby BA, Litonjua AA. Comprehensive genetic assessment of a functional TLR9 promoter polymorphism: no replicable association with asthma or asthma-related phenotypes.BMC Med Genet 2011; $12: 26$.

35. Kikuchi K, Lian ZX, Kimura Y, Selmi C, Yang GX, Gordon SC, Invernizzi P, Podda M, Coppel RL, Ansari AA, Ikehara S, Miyakawa H, Gershwin ME. Genetic polymorphisms of toll-like receptor 9 influence the immune response to $\mathrm{CpG}$ and contribute to hyper-IgM in primary biliary cirrhosis. J Autoimmun 2005; $24: 347-52$.

36. Chuang TH, Ulevitch RJ. Cloning and characterization of a sub-family of human toll-like receptors: hTLR7, hTLR8 and hTLR9. Eur Cytokine Netw 2000; 11 :372-8.

37. Lai ZZ, Ni Z, Pan XL, Song L. Toll-like receptor 9 (TLR9) gene polymorphisms associated with increased susceptibility of human papillomavirus-16 infection in patients with cervical cancer. J Int Med Res 2013; 41 :1027-36.

38. Tian S, Zhang L, Yang T, Wei X, Zhang L, Yu Y, Li Y, Cao D, Yang X. The Associations between TollLike Receptor 9 Gene Polymorphisms and Cervical Cancer Susceptibility. Mediators Inflamm 2018;2018 :9127146.

\section{Figure legends :}

Figure (1) : Relation between TLR3.rs3775290 (c.1377C/T) genotype and total HCV-specific CMI response among total subjects (A), seronegative, aviraemic subjects (B), seronegativeviraemic subjects (C), spontaneously resolved subjects (D), and chronic HCV patients (E). Data are shown as scatter plots. For each subject, the total cumulative number of HCV-specific IFN- $\gamma$ spot-forming cells (SFC) per $10^{6} \mathrm{PBMCs}$ is shown for responding and non-responding healthcare workers (HCWs) and patients. Error bars represent the SEM.

Figure (2) : Relationship betweenTLR9.rs5743836 (-1237T-C) genotype and total HCV-specific CMI response among the total subjects (A), seronegative aviraemic subjects (B), seronega- 
tiveviraemic subjects (C), spontaneously resolved subjects (D), and chronic HCV patients (E). Data are shown as scatter plots. For each subject, the total cumulative number of HCV-specific IFN- $\gamma$ spot-forming cells (SFC) per $10^{6} \mathrm{PBMCs}$ is shown for responding and non-responding healthcare workers (HCWs) and patients. Error bars represent the SEM.

Figure (3): Relationship between TLR9.rs352140 (G2848A) genotype and total HCV-specific CMI response among the total subjects (A) seronegative, aviraemic subjects (B) seronegative,viraemic subjects (C) spontaneously resolved subjects (D) andchronic HCV patients (E). Data are shown as scatter plots. For each subject, the total cumulative number of HCV-specific IFN- $\gamma$ spot-forming cells (SFC) per $10^{6}$ PBMCs is shown for responding and non-responding HCWs and patients. Error bars represent the SEM.

(A) Total subjects
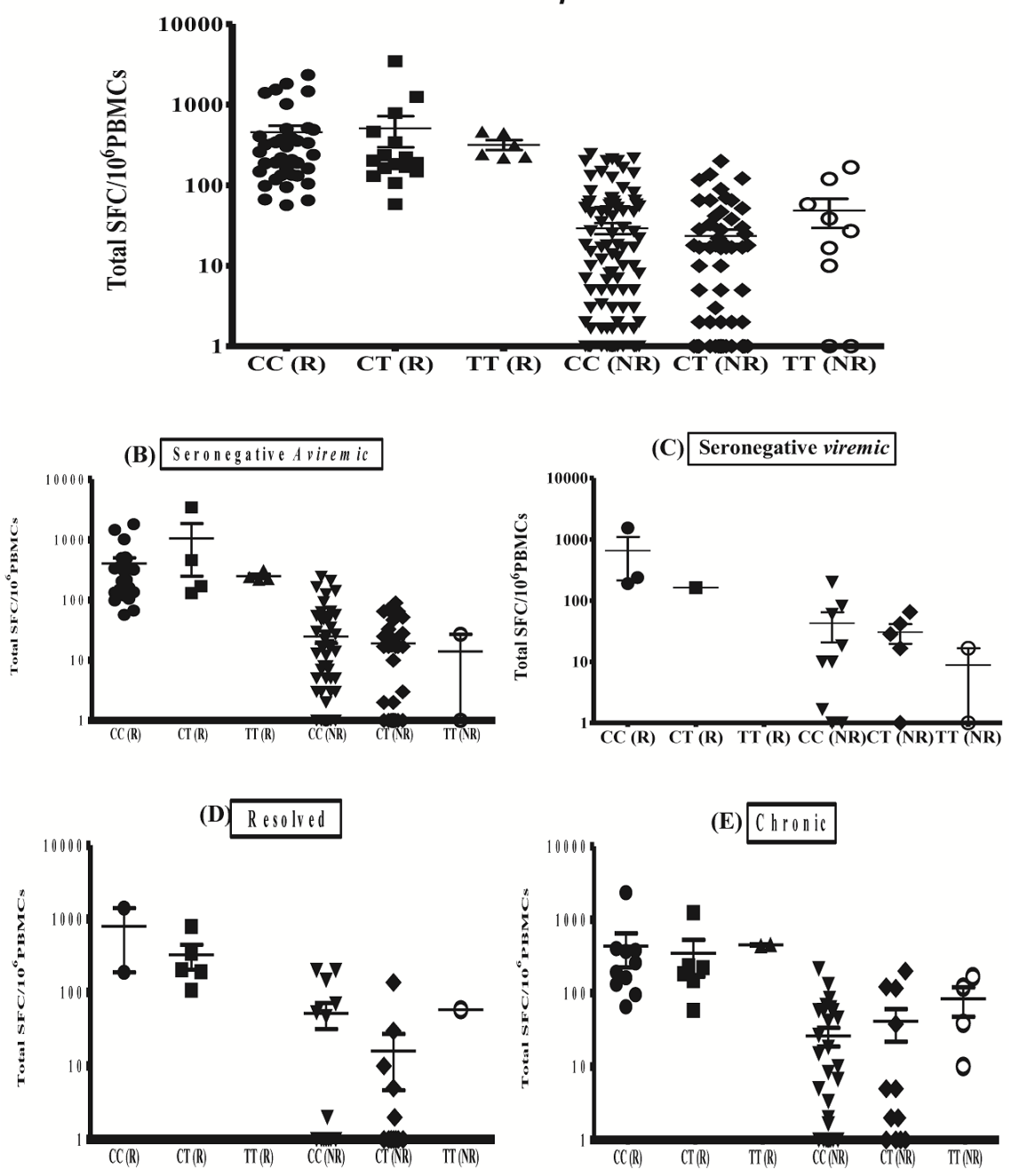

Figure 1: 

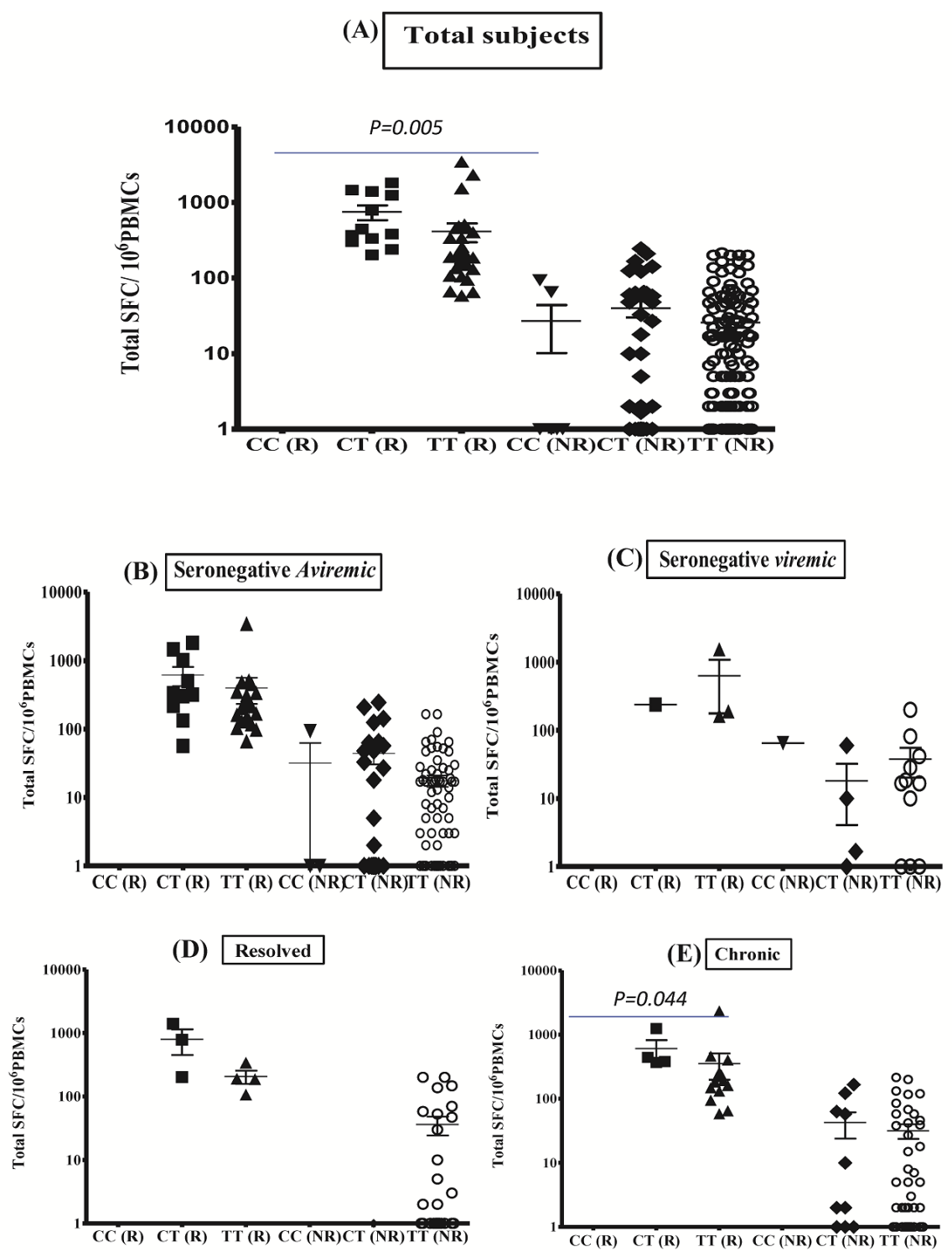

Figure 2: 

(A) Total subjects
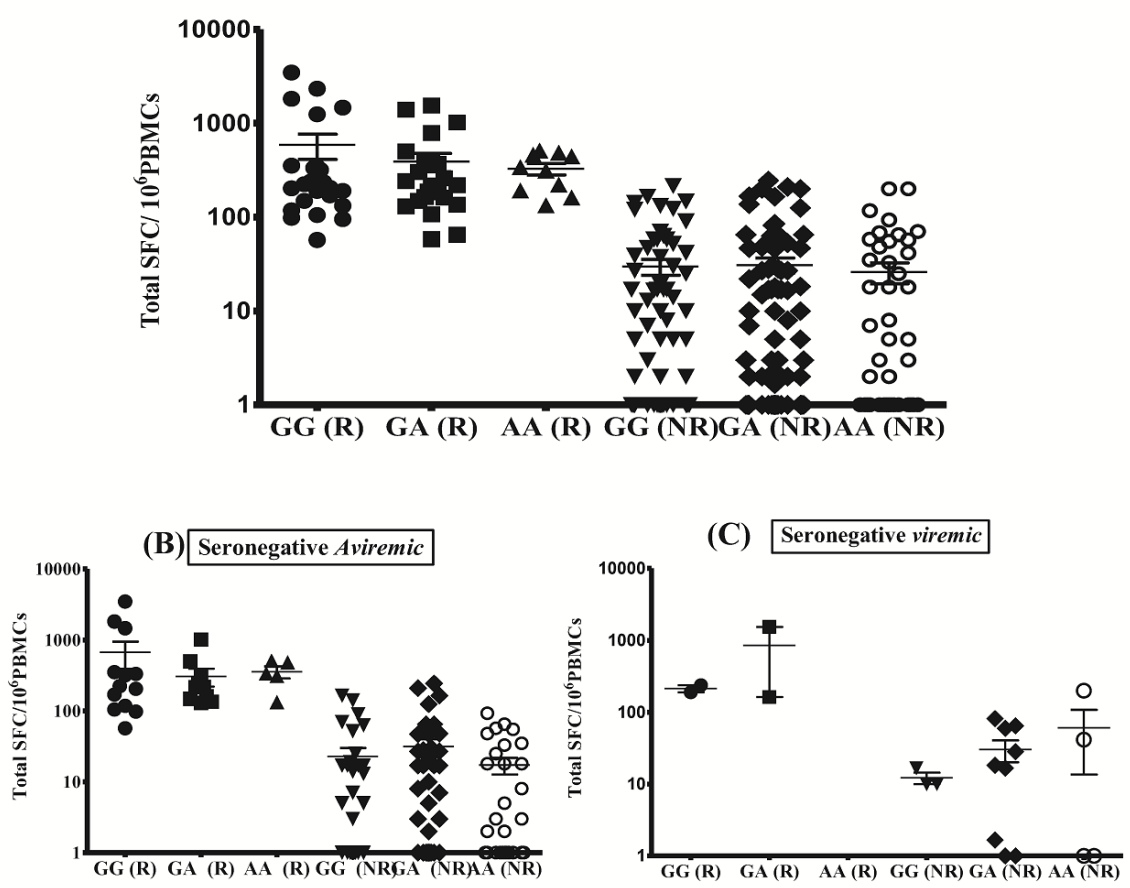

(D) Resolved

(E) Chronic
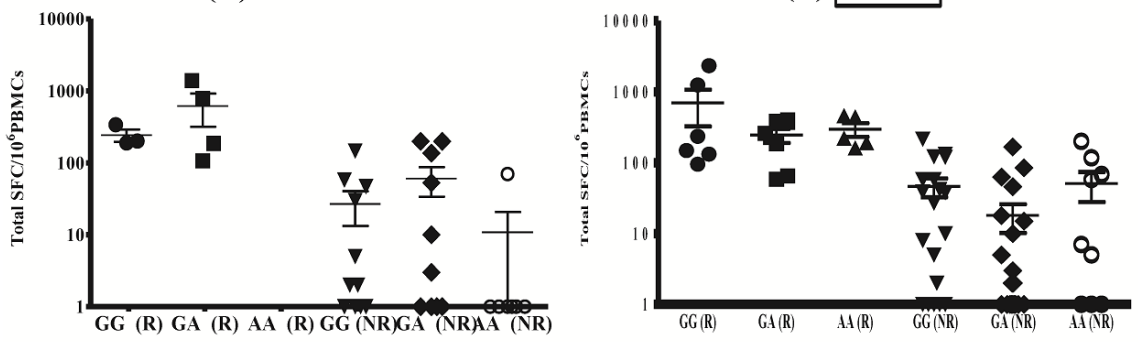

Figure 3: 